\title{
DETERMINACIÓN DE CONTAMINANTES ORGÁNICOS PERSISTENTES EN LECHE DE VACA DE PRODUCCIÓN NACIONAL POR MEDIO DE LA TÉCNICA DE CROMATOGRAFÍA DE GASES ACOPLADA A ESPECTROMETRÍA DE MASAS (GC/MS)
}

\section{Determination of persistent organic pollutants in cow's milk of national production by using the gas chromatography-mass spectrometry (GC/MS) technique}

\section{Camila Marranzini}

Laboratorio de la Dirección General de Aduanas, Santo

Domingo, República Dominicana.

ORCID: 0000-0003-3523-3915

Correo-e: cmmarranzini@gmail.com

\section{Lizaira Bello}

Instituto Tecnológico de Santo Domingo (INTEC),

Santo Domingo, República Dominicana.

ORCID: 000-0002-0258-3807

Correo-e: lizaira.bello@intec,edu.do

\section{Alejandro Vallejo Degaudenzi}

Instituto de Medicina Tropical y Salud Global, Santo

Domingo, República Dominicana.

ORCID: 0000-0003-2057-2332

Correo-e: a.vallejo@unibe.edo.do

\author{
Aris Mendis Gómez \\ Laboratorio de la Dirección General de Aduanas, Santo \\ Domingo, República Dominicana. \\ ORCID: 0000-0003-1045-5570 \\ Correo-e: e.echavarria@aduanas.gob.do \\ Enmanuel Echavarría \\ Laboratorio de la Dirección General de Aduanas, Santo \\ Domingo, República Dominicana. \\ ORCID: 0000-0002-3891-9456 \\ Correo-e: e.echavarria@aduanas.gob.do
}

Recibido: 13-X-2020 • Aprobado: 20-XI-2020

Cómo citar: Marranzini, C., Bello, L., Vallejo Degaudenzi, A., Gómez, A. M., \& Echavarría, E. (2020). Determinación de contaminantes orgánicos persistentes en leche de vaca de producción nacional por medio de la técnica de cromatografía de gases acoplada a espectrometría de masas (GC/MS). Ciencia, Ambiente y Clima, 3(2), 5-18. Doi: https://doi.org/10.22206/cac.2020.v3i2.pp5-18

\section{Resumen}

Los contaminantes orgánicos persistentes (COP) son sustancias químicas orgánicas capaces de persistir en el ambiente, transportase a larga distancia, bioacumularse y biomagnificarse en los ecosistemas. Los efectos nocivos de estos compuestos sobre el medio ambiente y la salud de los seres vivos son preocupantes. En particular, los seres humanos pueden entrar en contacto con los COP al consumir alimentos contaminados de origen animal con un alto contenido en grasa. En la República Dominicana estos compuestos se han utilizado y generado ampliamente. Sin embargo, se desconoce cuáles son los niveles de exposición

\begin{abstract}
Persistent organic pollutants (POPs) are organic chemicals capable of persisting in the environment, traveling long distances, bioaccumulate and biomagnify in ecosystems. The harmful effects of these compounds on the environment and the health of living beings are alarming. In particular, humans can be exposed POPs by consuming contaminated fatty animal foods. In the Dominican Republic these compounds have been widely used and generated. However, the degree of POPs exposure to which the population is exposed is unknown. Therefore, this study aimed to determine the presence of 34 POPs in five main brands
\end{abstract}


de COP a los que está expuesta la población. Por tanto, este estudio tuvo como objetivo determinar la presencia de 34 COP en cinco principales marcas de leche de vaca de producción nacional. La preparación de las muestras se realizó mediante el método de extracción QuEChERS y la técnica analítica utilizada fue la GC/MS. Los resultados obtenidos indicaron que no hay presencia de los COP evaluados en ninguna de las muestras de leche de vaca, lo cual sugiere que su consumo no representa una amenaza para la salud de los consumidores. Además, este estudio aporta al conocimiento sobre la evaluación de los COP en la República Dominicana.

Palabras claves: contaminante; leche; República Dominicana; análisis cromatográfico; química agrícola.

\section{Introducción}

Las sustancias químicas conocidas como contaminantes orgánicos persistentes (COP) son el centro de atención internacional a causa de la creciente evidencia científica que sustenta que estos pueden provocar afecciones como el cáncer, daños en los sistemas nerviosos central y periférico, enfermedades del sistema inmune, desórdenes reproductivos, trastornos metabólicos y alteraciones en el desarrollo normal de bebés y niños (Secretariat of the Stockholm Convention, 2019). Además, estos compuestos son capaces de persistir en el medio ambiente, transportarse a larga distancia a través de la atmósfera, bioacumularse en tejidos humanos y animales y biomagnificar sus concentraciones en las cadenas alimentarias (World Health Organization (WHO) Persistent organic pollutants (POPs), 2014). Los COP han sido ampliamente utilizados en diversas actividades humanas; como plaguicidas, en distintos procesos farmacéuticos, y en la generación de productos y subproductos químicos en varias industrias. En particular, los COP incluyen diferentes tipos de especies químicas tales como, los pesticidas organoclorados, las dibenzo-dioxinas policloradas (PCDD), los dibenzo-furanos policlorados (PCDF) y los bifenilos policlorados (PCB) (Secretariat of the Stockholm Convention, 2017). of nationally produced cow's milk. The preparation of the samples was carried out using the QuEChERS extraction method and the analytical technique used was GC/MS. The results obtained indicate that there is no presence of the POPs evaluated in any of the cow's milk samples. These findings suggest that the consumption of this product does not represent a threat to the health of consumers. Furthermore, this study contributes to the knowledge on the evaluation of POPs in the Dominican Republic.

Keywords: Pollutant; milk; Dominican Republic; chromatographic analysis; agricultural chemistry.

En consecuencia, se ha generado una exposición sostenida de muchas especies, incluidos los seres humanos, durante períodos de tiempo que abarcan generaciones. Con el fin de mitigar los efectos adversos que producen estas sustancias fue creado el tratado mundial conocido como el Convenio de Estocolmo en el que los países participantes, incluyendo la República Dominicana, acordaron eliminar o reducir la producción, el uso y la liberación de los COP. Distintas actividades, tales como el uso de pesticidas organoclorados, la quema de desechos y el uso de transformadores y capacitadores eléctricos han contribuido a la dispersión de los COP en el país (Sbriz, Aquino, Rodriguez, Fowler, \& Sericano, 1998; Secretaría de Estado de Medio Ambiente y Recursos Naturales (SEMARENA), 2008).

En concreto, la exposición de los seres humanos a los COP ocurre principalmente a través de alimentos contaminados, en especial de aquellos de origen animal con un alto contenido en grasa (Lee et al., 2006). En consecuencia, organizaciones internacionales como la Agencia de Protección Ambiental de los Estados Unidos (USEPA), la Unión Europea (UE) y el Codex Alimentarius de la Organización de las Naciones Unidas para la Alimentación y la Agricultura (FAO por sus siglas en inglés) han creado 
estándares donde se establecen los límites máximos de residuos (LMR) de COP permitidos en los alimentos (Codex Alimentarius of Food and Agriculture Organization of the United Nations/World Health Organization (FAO/WHO), 2020; European Commission, 2020; United States Environmental Protection Agency (US EPA), 2020). Debido a la necesidad de estudiar los efectos adversos en los seres humanos producidos por el consumo de alimentos contaminados con dichas sustancias, diversas técnicas analíticas han sido utilizadas para su detección e identificación (Fernández-Alba \& García-Reyes, 2008; Hernández, Grimalt, Pozo, \& Sancho, 2009; Lacina, Urbanova, Poustka, \& Hajslova, 2010; Polgár et al., 2012). Sin embargo, el método de extracción rápido, fácil, económico, eficaz, robusto y seguro (QuEChERS, por sus siglas en inglés) y la técnica analítica de cromatografía de gases acoplada a espectrometría de masa (GC/MS, por sus siglas en inglés) son las herramientas más empleadas en la actualidad debido a sus destacadas ventajas de flexibilidad, rapidez, economía, facilidad, eficiencia y robustez, alta sensibilidad, exactitud y versatilidad (Chen, Lin, Dang, \& Puschner, 2017; Jawaid, Talpur, Nizamani, Khaskheli, \& Afridi, 2016; Rahman et al., 2018; Selvi, Paramasivam, Rajathi, \& Chandrasekaran, 2012). En particular, abundantes estudios científicos evalúan el contenido de COP en productos lácteos como la leche e incluso se han detectado concentraciones por encima de los LMR establecidos internacionalmente (Battu, Singh, \& Kang, 2004; Chen et al., 2017; Dagnac, Garcia-Chao, Pulleiro, Garcia-Jares, \& Llompart, 2009; Fromberg, Granby, Højgård, Fagt, \& Larsen, 2011; Jawaid et al., 2016; Martins, Amaya Chávez, Waliszewski, Colín Cruz, \& García Fabila, 2013; Rahman et al., 2018; Rawash et al., 2018; Selvi et al., 2012).

Por el contrario, en la República Dominicana el número de investigaciones sobre la presencia de COP y sus efectos sobre la población son muy escasos y antiguos (Rodríguez, De Pratt, Peña, \& Beltré, 2006;
Sbriz et al., 1998; Subsecretaría de Estado de Recursos Naturales de la Secretaría de Estado de Agricultura (SEA-SURENA), 1999). Se cree que la liberación y el uso de estas sustancias tóxicas ha disminuido al pasar de los años debido a la implementación de normativas que regulan el manejo de los COP. No obstante, se desconoce la situación real de los COP en el país. Es por ello que, este estudio tuvo el objetivo de determinar la presencia de $34 \mathrm{COP}$ en cinco marcas de leche de vaca producidas nacionalmente con la finalidad de obtener información certera y confiable a través de la aplicación de técnicas analíticas sensibles y eficaces sobre el grado de exposición a los COP en alimentos que presenta la sociedad dominicana.

\section{Materiales y métodos}

\section{Reactivos y muestras}

Esta investigación determinó en las muestras de leche de vaca la presencia de los siguientes COP: aldrín, clordano, decabromobifenil, diclorodifenildicloroetano (DDD), diclorodifenildicloroetileno (DDE), diclorodifeniltricloroetano (DDT), dieldrín, endosulfán, endrín, heptacloro, hexabromobifenilo, hexabromociclododecano (HBCD), hexaclorobenceno, hexaclorociclohexano $(\mathrm{HCH})$, lindano (gamma-HCH), mirex, toxafeno y los siguientes congéneres de PCB: 2,3,3',4,5'-pentaclorobifenilo (PCB 122), 2,2',3,3',4,4',5-heptaclorobifenilo (PCB 170), 2,3',4,4',6-pentaclorobifenilo (PCB 119), 3,3',4,4'-tetraclorobifenilo (PCB 77), 2,3,4,4',5-pentaclorobifenolo(PCB114),2,2',3,4,4', ', -hexaclorobifenilo (PCB 138), 2,2',4,5,5'-pentaclorobifenilo (PCB 101), 2,2',4,4',5, 5'-hexaclorobifenilo (PCB 153), 2,2', 5, 5'-tetraclorobifenilo (PCB 52), 2,2',3,4,4'5, ''-heptaclorobifenilo (PCB 180), 2,4,4'-triclorobifenilo (PCB 28), 2,3,3',4,4',5 -hexaclorobifenilo (PCB 156), 2,3',4,4',5,5'-hexaclorobifenilo(PCB 167), 2,3,3',4,4'-pentaclorobifenilo (PCB 105), 2,3,3',4,4' 5, 5' -heptaclorobifenilo (PCB 189), 3,4,4',5-tetraclobifenilo (PCB 81) 
y 3,3',4,4',5,5'-hexaclorobifenilo (PCB 169). El método creado por la Asociación de Químicos Analíticos Oficiales (AOAC) denominado AOAC 2007.01 fue utilizado como base de la presente experimentación (Association of Official Analytical Chemists, 2007). Todos los reactivos y materiales utilizados eran certificados y de alta pureza, grado LC/MS, óptima y grado pesticida de la marca Fisher. En adición, fueron seleccionadas como muestras para el desarrollo de este estudio cinco marcas de leche de vaca con un contenido de grasa de $3 \%$ (leche entera) producidas en la República Domini- cana. La metodología se llevó a cabo a través de las siguientes etapas: recolección de la muestra, preparación de la muestra y la técnica analítica.

Durante los meses de mayo a julio del año 2019, fueron seleccionadas cinco marcas distintas de leche entera de vaca de producción nacional comercializadas en los principales supermercados del Distrito Nacional. Tres lotes o producciones de cada marca de leche entera fueron seleccionados al azar. Los análisis de cada uno de los 15 lotes fueron repetidos tres veces, con un total de 45 muestras (ver figura 1).
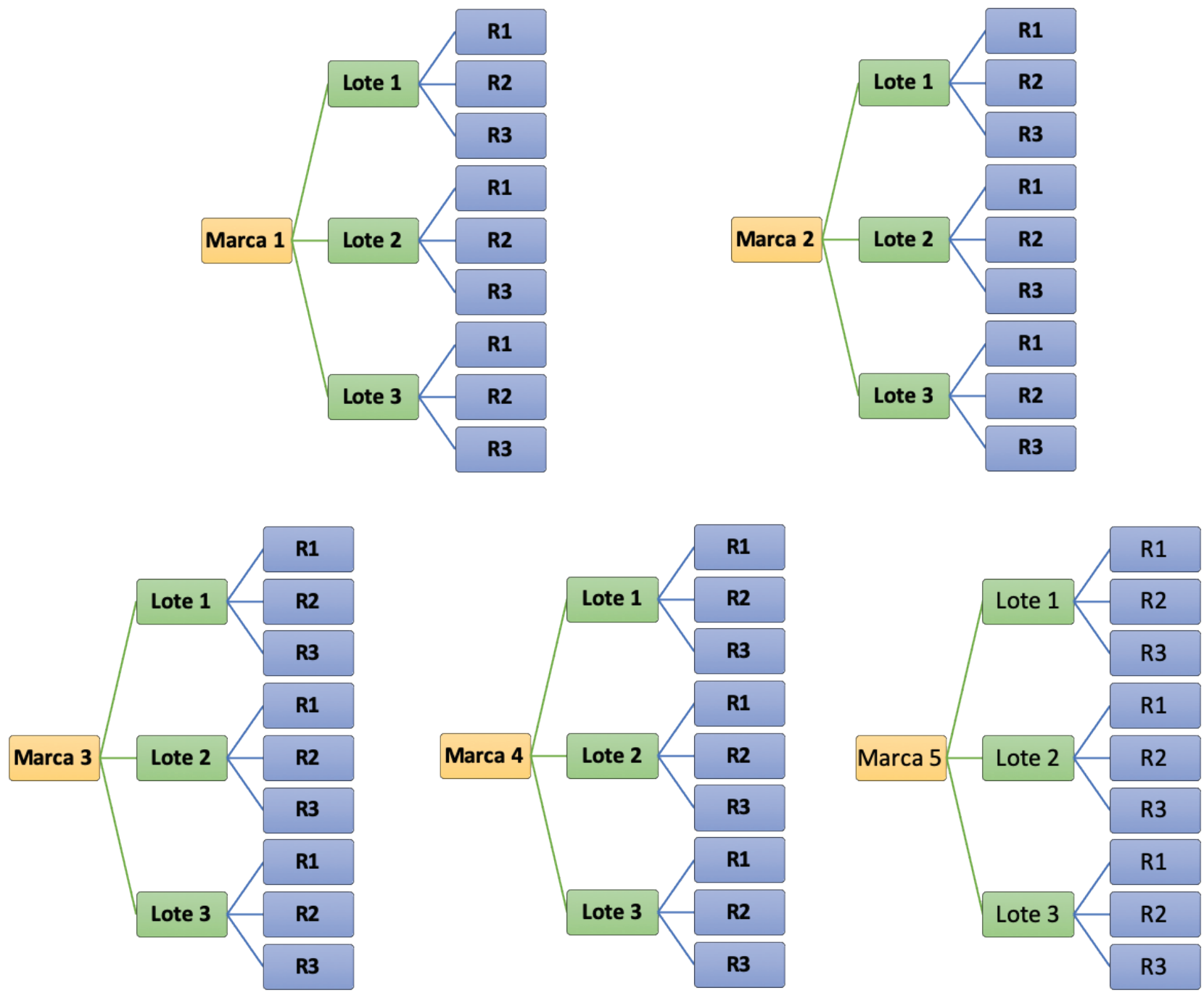

Figura 1. Esquema del diseño experimental

Fuente: elaboración propia.

8 | Ciencia, Ambiente y Clima 2020; 3(2, julio-diciembre): 5-18 


\section{Preparación de la muestra}

La preparación de la muestra se desarrolló en tres partes: extracción, purificación y concentración (ver figura 2). En primer lugar, se vertieron $15 \mathrm{~mL}$ de leche en tubos de extracción y se agregaron $6 \mathrm{~g}$ de sulfato de magnesio $\left(\mathrm{MgSO}_{4}\right)$ y $1.5 \mathrm{~g}$ de acetato de sodio $(\mathrm{NaOAc})$, seguido de la adición de $15 \mathrm{~mL}$ de una solución de acetonitrilo $\left(\mathrm{C}_{2} \mathrm{H}_{3} \mathrm{~N}\right)$ con $1 \%$ de ácido acético $\left(\mathrm{CH}_{3} \mathrm{CO}_{2} \mathrm{H}\right)$. Se agitó vigorosamente por un minuto, y se centrifugó a 3,900 rpm por $8 \mathrm{~min}$.

Luego, $8 \mathrm{~mL}$ del sobrenadante fueron transferidos a los tubos de extracción de fase sólida disper- siva (d-SPE) que contenían $150 \mathrm{mg}$ de sulfato de magnesio $\left(\mathrm{MgSO}_{4}\right), 50 \mathrm{mg}$ de etilendiamina-N-propilo (amina primaria/secundaria, PSA), $50 \mathrm{mg}$ de un sorbente C18 (sílice octadecil unido) y $50 \mathrm{mg}$ de carbón activado grafitado (GCB). Después, fue agitado por un minuto y centrifugado a 3,900 rpm durante $8 \mathrm{~min}$.

Posteriormente, la fase superior separada fue concentrada, y el solvente fue intercambiado por tolueno $\left(\mathrm{C}_{6} \mathrm{H}_{5} \mathrm{CH}_{3}\right)$. Para esto, se calentó el extracto final a una temperatura constante de $60^{\circ} \mathrm{C}$ hasta la evaporación del solvente y luego se agregó $1 \mathrm{~mL}$ de tolueno. Finalmente, esa solución fue transferida a viales de GC de $1.5 \mathrm{~mL}$.

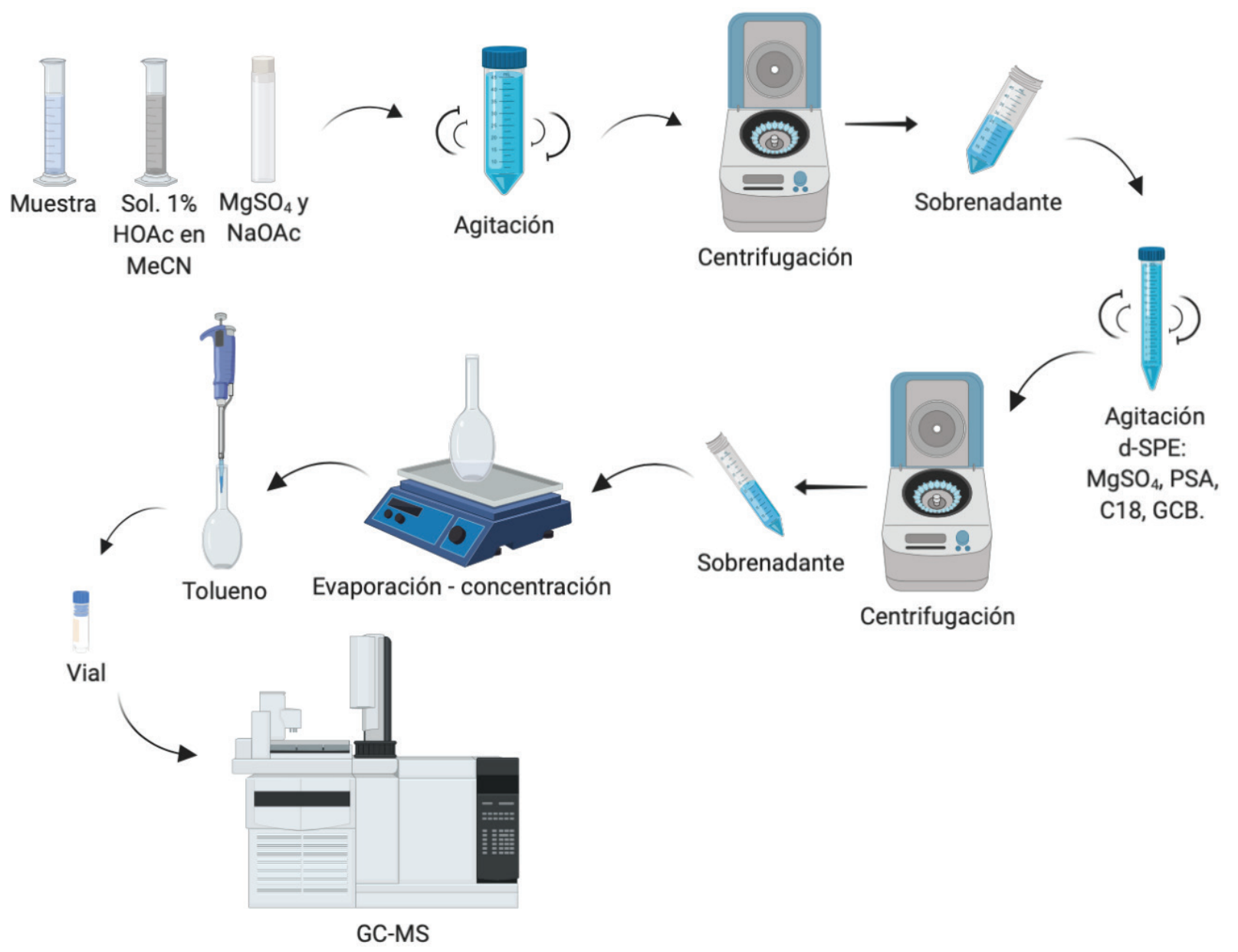

Figura 2. Flujo de trabajo para la preparación de la muestra

Fuente: elaboración propia. 


\section{Técnica analítica}

La determinación de la presencia de COP evaluados en las muestras de leche se llevó a cabo por la técnica GC/MS que es la recomendada por las normas, directrices y códigos de prácticas internacionales sobre alimentos del Codex Alimentarius para la identificación y confirmación de residuos de COP en los alimentos (Codex Committee on Pesticide Residues (CCPR), 2017). El equipo utilizado fue el GC/MS Clarus SQ $8 \mathrm{C}$ de la marca Perkin Elmer. Se inyectó, en modo split, $1 \mu \mathrm{L}$ del extracto final en la columna Elite 5MS marca Perkin Elmer de espesor de la película de 0.25 $\mu \mathrm{m}$, diámetro interior de $0.25 \mathrm{~mm}$, longitud de $60 \mathrm{~m}$ y fase de 1,4-bis (dimetilsiloxi) fenileno dimetilpolisiloxano. El hidrógeno fue utilizado como gas portador y se mantuvo con un flujo constante de $1.5 \mathrm{~mL} / \mathrm{min}$. La rampa de temperatura del horno de la columna se inició a partir de $90{ }^{\circ} \mathrm{C}$ con un incremento de $3{ }^{\circ} \mathrm{C} / \mathrm{min}$ hasta alcanzar $320^{\circ} \mathrm{C}$. El inyector se mantuvo a una temperatura constante de $250^{\circ} \mathrm{C}$, para un tiempo total de corrida de 75 minutos.

El detector MS fue utilizado en el modo de escaneo completo para evaluar el rango de COP posible en GC/MS, seleccionando un rango de masas que va desde los 50 a los $550 \mathrm{~m} / \mathrm{z}$, con una energía de colisión del electrón de $70 \mathrm{eV}$. La fuente de ionización utilizada fue la de impacto electrónico. La línea de transferencia y la fuente de ionización se mantuvieron a una temperatura de $300{ }^{\circ} \mathrm{C}$ y $320{ }^{\circ} \mathrm{C}$, respectivamente.

\section{Estándares de referencia}

Fueron utilizados los compuestos naftaleno-d8 y acenafteno-d10 como estándar interno con una concentración de 40 ppm.

A modo de control de calidad, se preparó una solución que contenía una mezcla de estándares externos de referencia de los COP evaluados de marca Sigma Aldrich con una pureza entre $95.8 \%$ y $99.8 \%$, y estos fueron estudiados bajo las mismas condiciones cromatográficas anteriormente descritas. Además, presentaron un límite de detección (LOD) de $0.010 \mathrm{ppm}$. Posteriormente, los cromatogramas y espectros de masas obtenidos de estos estándares externos fueron comparados con los que resultaron de las muestras.

\section{Resultados y discusión}

\section{Selección de la muestra}

La selección de leche procesada como muestra para el desarrollo de este estudio fue satisfactoria, debido a que los tratamientos usuales en la leche para consumo humano tales como la pasteurización, la esterilización y el procesamiento UHT no causan ningún efecto apreciable en el contenido de residuos de COP en esta matriz (Deiana \& Fatichenti, 1992). Además, la evaporación e intercambio del solvente contribuyeron a la reducción o a la eliminación de interferencias y a la concentración de los analitos objetivo. 


\section{Método de extracción QuEChERS}

El uso de sulfato de magnesio $\left(\mathrm{MgSO}_{4}\right)$ y acetato de sodio $(\mathrm{NaOAc})$ en el método de extracción QuEChERS fueron utilizados satisfactoriamente para remover el agua presente en las muestras. También, el uso de etilendiamina-N-propilo (amina primaria/ secundaria, PSA) fue exitoso para eliminar ácidos grasos, otros ácidos orgánicos y azúcares, al igual que el uso del sorbente C18 (sílice octadecil unido) con el fin de remover interferencias no polares contenidas en las muestras.

\section{Técnica analítica GC/MS}

La utilización de la técnica analítica de GC/MS permitió la completa separación e identificación de todos los componentes presentes en las muestras, arrojando cromatogramas y espectros de masas característicos (ver figuras 3 y 4). Luego de realizar los análisis por medio de dicha técnica, en este estudio no se detectó la presencia de ninguno de los COP evaluados en ninguna de las muestras de leche de vaca.

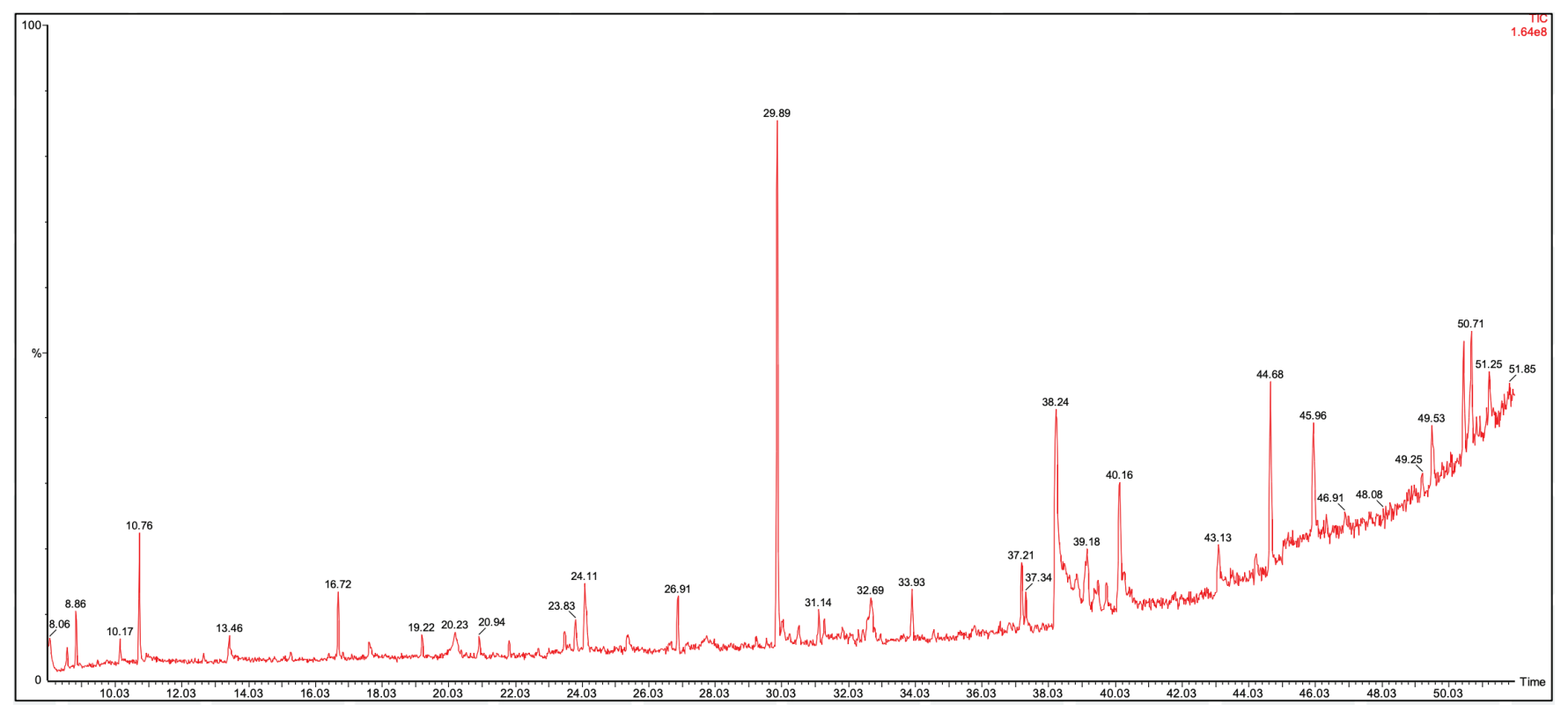

Figura 3. Cromatograma de una muestra de leche

Fuente: elaboración propia. 


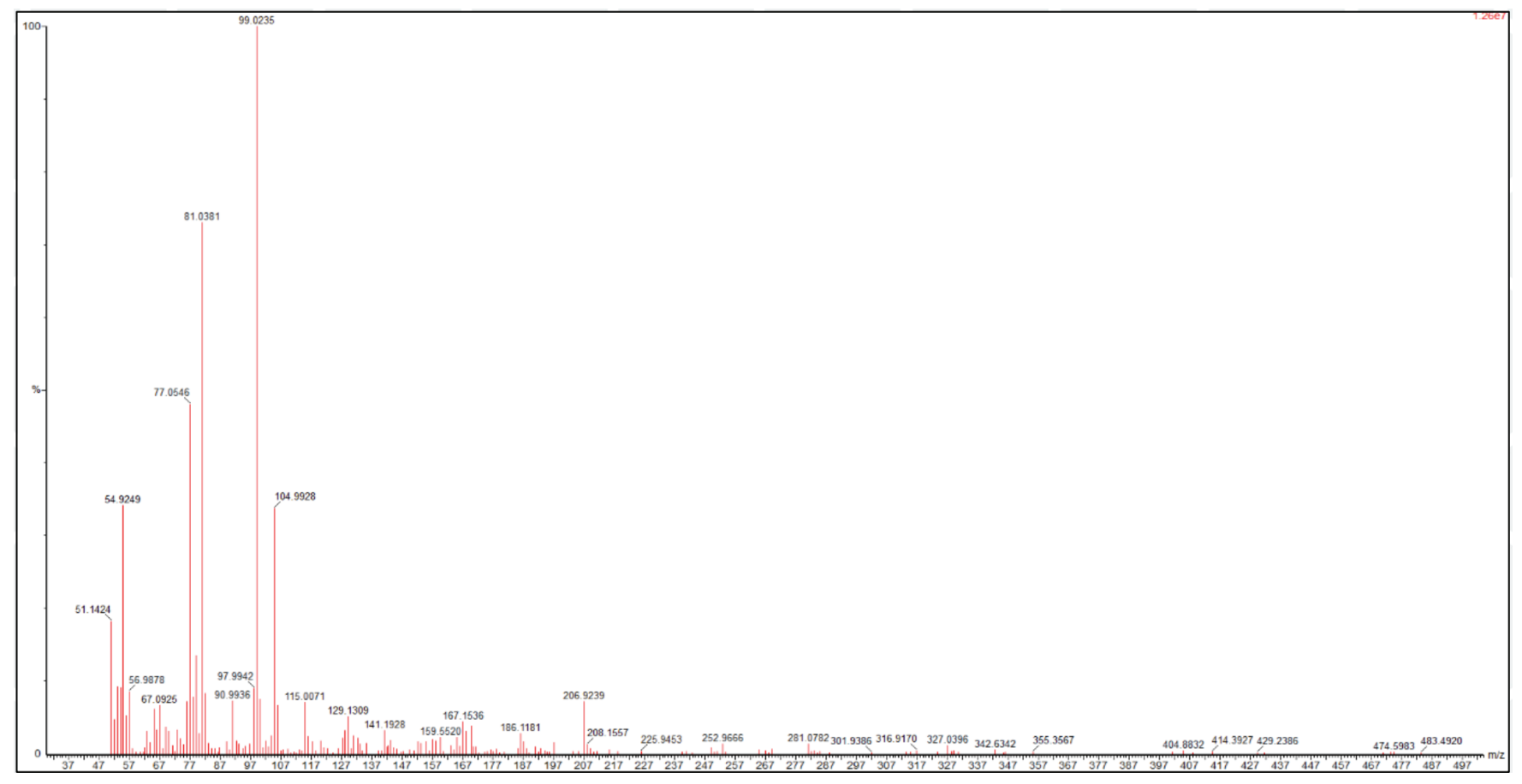

Figura 4. Espectro de masas de un componente de una muestra de leche

Fuente: elaboración propia.

\section{Confirmación de resultados}

Los espectros de masas obtenidos fueron corroborados con la biblioteca espectral de masas NIST y el registro de datos espectrales de masas Wiley (National Institute of Standards and Technology, 2018; Wiley, 2016), confirmando la ausencia de los COP evaluados en las muestras.

En adición, la solución de la mezcla de estándares de referencia de los COP evaluados fue analizada bajo las mismas condiciones que las muestras y esta mostró tiempos de retención (véase figura 5), espectros de masas (véase figura 6) e iones de fragmentación (véase tabla 1) específicos para cada compuesto. Dichos datos fueron comparados con los respectivos cromatogramas y espectros de masas obtenidos de las muestras y estos no coincidieron, confirmando que no había presencia de ninguno de los COP evaluados en las muestras de leche de vaca. 


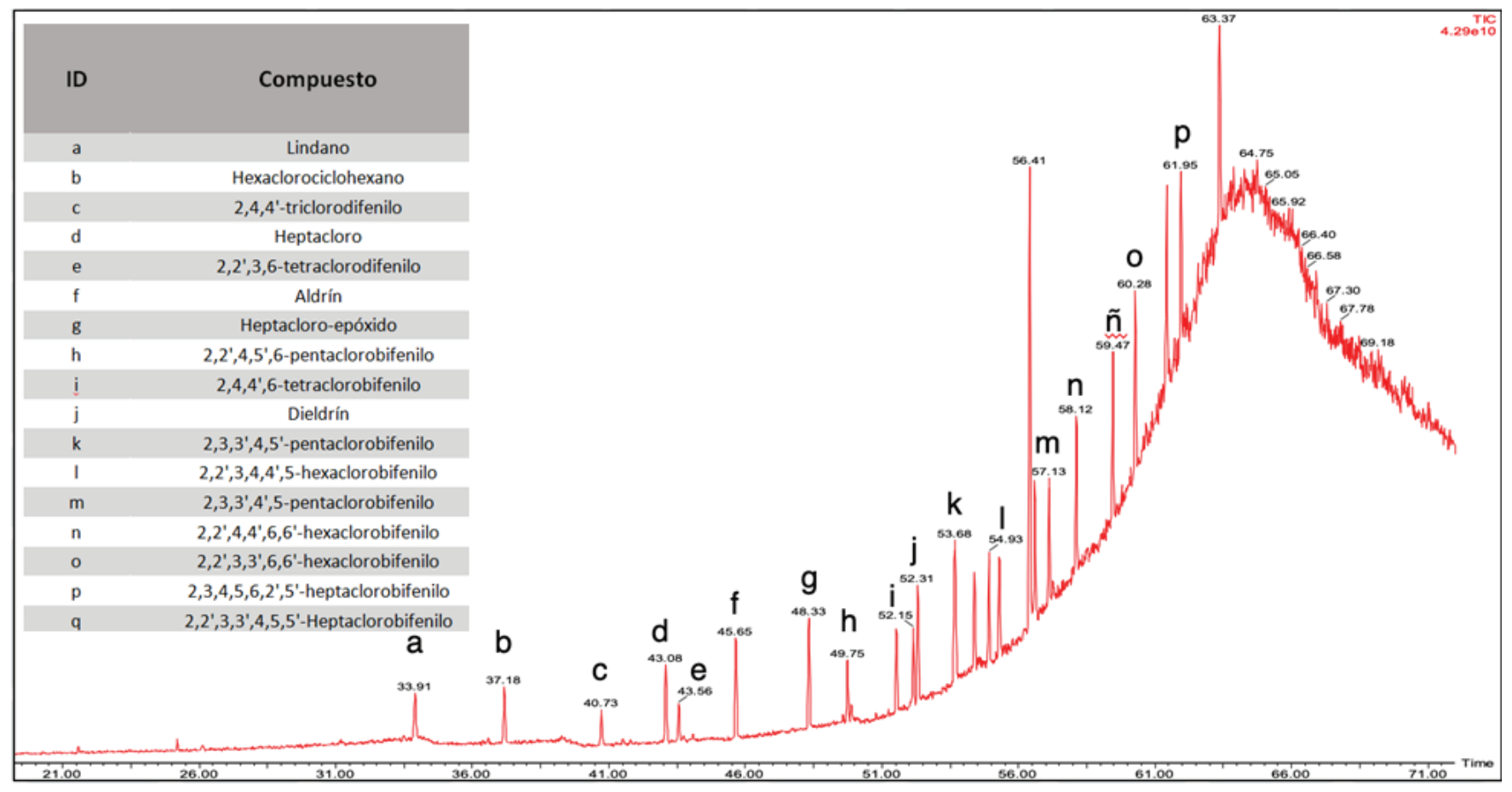

Figura 5. Cromatograma de una mezcla de estándares externos de referencia de algunos de los COP evaluados Fuente: elaboración propia.

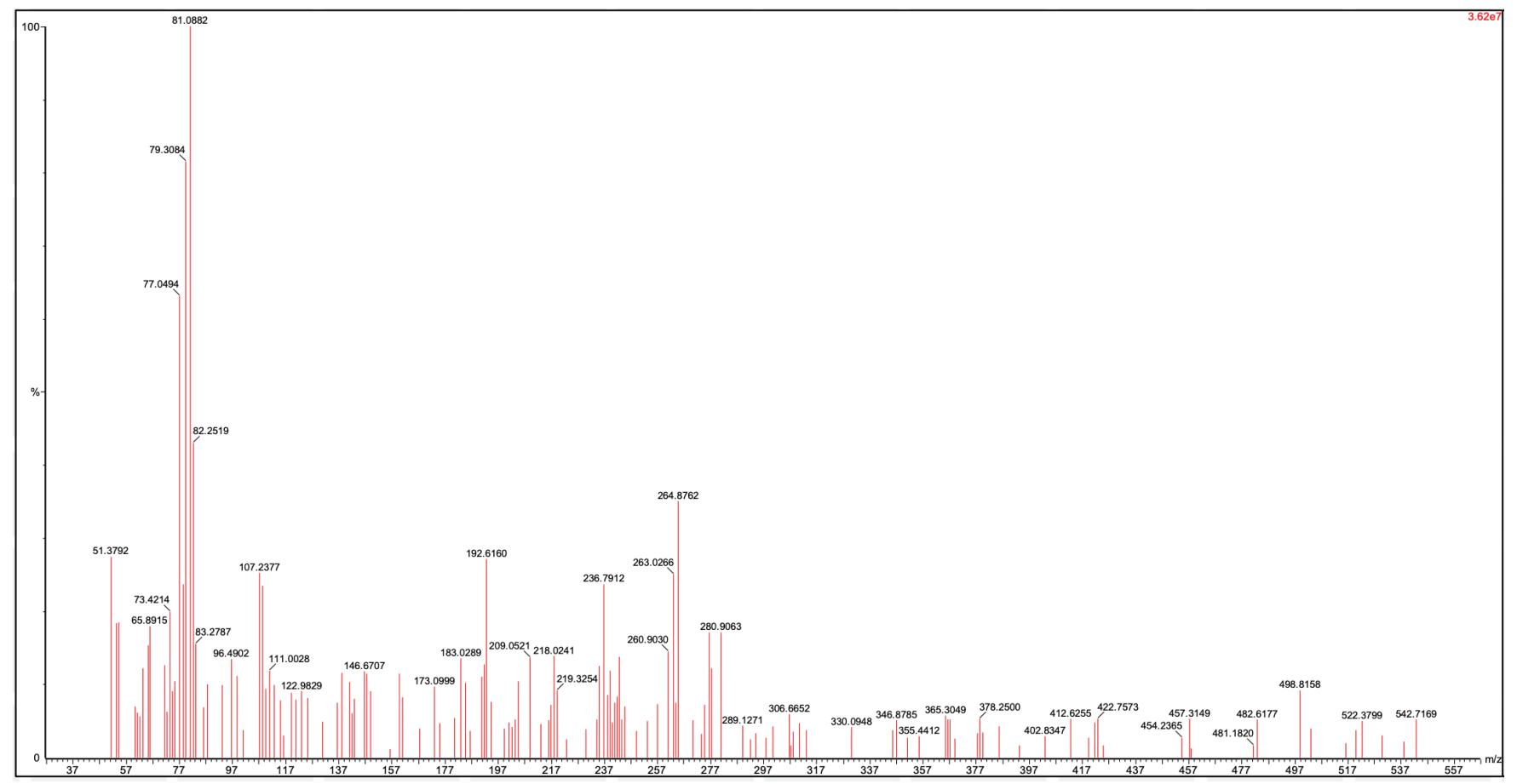

Figura 6. Espectro de masas del estándar externo de referencia dieldrín

Fuente: elaboración propia. 
Tabla 1. Valores masa/carga $(\mathrm{m} / \mathrm{z})$ de la fragmentación de iones de los estándares externos

\begin{tabular}{|c|c|c|c|c|}
\hline Compuesto & $\begin{array}{c}\text { Ion } \\
\text { molecular } \\
(\mathbf{m} / \mathbf{z})\end{array}$ & $\begin{array}{c}\text { Ion de } \\
\text { fragmentación } \\
\text { primario }(\mathrm{m} / \mathrm{z})\end{array}$ & $\begin{array}{c}\text { Ion de } \\
\text { fragmentación } \\
\text { secundario } \\
(\mathrm{m} / \mathrm{z})\end{array}$ & $\begin{array}{c}\text { Ion de } \\
\text { fragmentación } \\
\text { terciario }(\mathrm{m} / \mathrm{z})\end{array}$ \\
\hline Lindano & 288 & 181 & 219 & 109 \\
\hline Hexaclorociclohexano & 288 & 183 & 181 & 219 \\
\hline 2,4,4'-triclorodifenilo & 256 & 256 & 258 & 186 \\
\hline Heptacloro & 370 & 100 & 272 & 274 \\
\hline 2,2',3,6-tetraclorodifenilo & 290 & 292 & 220 & 290 \\
\hline Aldrín & 362 & 66 & 263 & 265 \\
\hline Heptacloro-epóxido & 386 & 81 & 353 & 355 \\
\hline 2,2',4,5',6-pentaclorobifenilo & 324 & 326 & 324 & 328 \\
\hline 2,4,4',6-tetraclorobifenilo & 290 & 292 & 290 & 220 \\
\hline Dieldrín & 378 & 79 & 81 & 82 \\
\hline 2,3,3',4,5'-pentaclorobifenilo & 324 & 326 & 324 & 328 \\
\hline 2,2',3,4,4’,5-hexaclorobifenilo & 358 & 360 & 362 & 290 \\
\hline 2,3,3',4',5-pentaclorobifenilo & 324 & 326 & 324 & 254 \\
\hline 2,2',4,4',6,6'-hexaclorobifenilo & 360 & 360 & 362 & 145 \\
\hline 2,2',3,3',6,6'-hexaclorobifenilo & 360 & 360 & 362 & 358 \\
\hline 2,3,4,5,6,2',5'-heptaclorobifenilo & 392 & 394 & 396 & 324 \\
\hline 2,2',3,3',4,5,5'-Heptaclorobifenilo & 392 & 396 & 394 & 324 \\
\hline
\end{tabular}

Fuente: elaboración propia.

14 | Ciencia, Ambiente y Clima 2020; 3(2, julio-diciembre): 5-18 


\section{Conclusiones}

En este trabajo se logró determinar que no hay presencia de los COP evaluados en ninguna de las muestras de leche de vaca de las principales marcas de producción nacional. Estos resultados pueden deberse a la reducción del uso y generación de los COP en el país, a raíz de las legalizaciones nacionales e internacionales que han sido implementadas con el fin de regular la aplicación de estos compuestos.

En adición, a diferencia de otros países de la región tales como Estados Unidos (Chen et al., 2017), Brasil (Ciscato et al., 2002), Argentina (Lorenzatti, Maitre, \&Lenardon, 2003), Colombia (Lans-Ceballos, Lombana Gomez, \& Pinedo-Hernández, 2018) y Chile (Muñoz, 2005) las muestras de leche de vaca producidas en la República Dominicana no suponen una fuente de exposición a los COP evaluados para las personas que consumen este producto. De igual forma, este estudio desempeña un papel muy importante en el aporte a las investigaciones sobre los niveles actuales de COP en la República Dominicana, ya que es el primer estudio de investigación sobre la determinación de los COP en leche de vaca producida y consumida en la República Dominicana.

\section{Recomendaciones}

Atendiendo a los resultados obtenidos en esta investigación, se recomienda realizar estudios con objetivos similares que evalúen otras sustancias clasificadas como COP, con el fin de tener una mejor estimación del contenido de COP en la leche de vaca producida en el país.

Asimismo, se sugiere ampliar el número de muestras, incluyendo otras marcas de leche producidas nacionalmente y con distinto contenido de grasa con el propósito de obtener resultados más completos y representativos.

Por último, se recomienda realizar estudios en otros alimentos y evaluar la presencia de los diferentes
COP, para así conocer la exposición real de los consumidores a los alimentos contaminados con COP producidos en la República Dominicana.

\section{Referencias}

Association of Official Analytical Chemists. (2007). AOAC Official Method 2007.01 Pesticide Residues in Foods by Acetonitrile Extraction and Partitioning with Magnesium Sulfate Gas Chromatography/Mass Spectrometry and Liquid Chromatography/Tandem Mass Spectrometry First Action 2007. Recuperado de http://www.eoma. aoac.org $/$ methods/info.asp?ID $=48938$

Battu, R. S., Singh, B., \& Kang, B. K. (2004). Contamination of liquid milk and butter with pesticide residues in the Ludhiana district of Punjab state, India. Ecotoxicology and Environmental Safety, 59(3), 324-331. Recuperado de https:// doi.org/10.1016/J.ECOENV.2003.08.017

Chen, X., Lin, Y., Dang, K., \& Puschner, B. (2017). Quantification of Polychlorinated Biphenyls and Polybrominated Diphenyl Ethers in Commercial Cows' Milk from California by Gas Chromatography-Triple Quadruple Mass Spectrometry. PloS One, 12(1), e0170129. Doi: https://doi.org/10.1371/journal.pone. 0170129

Codex Alimentarius of Food and Agriculture Organization of the United Nations/World Health Organization FAO/WHO. (2020). Maximum Residue Limit (MRL). Recuperado de http:// www.fao.org/fao-who-codexalimentarius/ codex-texts/dbs/pestres/glossary/en/

Dagnac, T., Garcia-Chao, M., Pulleiro, P., GarciaJares, C., \& Llompart, M. (2009). Dispersive solid-phase extraction followed by liquid chromatography-tandem mass spectrometry for the multi-residue analysis of pesticides in raw bovine milk. Journal of Chromatography A, 1216(18), 3702-3709. Doi: https://doi. org/10.1016/J.CHROMA.2009.02.048 
Deiana, P., \& Fatichenti, F. (1992). Pesticide residues in milk processing. Italian Journal of Food Science (Italy), 4(4), 229-245. Recuperado de http://agris.fao.org/agris-search/search.do?recordID=IT9362099

European Commission. (2020). EU Pesticides database. Recuperado de https://ec.europa.eu/ food/plant/pesticides/eu-pesticides-database/ public/?event=homepage\&language $=\mathrm{EN}$

Fernández-Alba, A. R., \& García-Reyes, J. F. (2008). Large-scale multi-residue methods for pesticides and their degradation products in food by advanced LC-MS. TrACTrends in Analytical Chemistry, 27(11), 973-990. Doi: https://doi. org/10.1016/J.TRAC.2008.09.009

Fromberg, A., Granby, K., Højgård, A., Fagt, S., \& Larsen, J. C. (2011). Estimation of dietary intake of $\mathrm{PCB}$ and organochlorine pesticides for children and adults. Food Chemistry, 125(4), 1179-1187. Doi: https://doi.org/10.1016/J. FOODCHEM.2010.10.025

Hernández, F., Grimalt, S., Pozo, Ó. J., \& Sancho, J. V. (2009). Use of ultra-high-pressure liquid chromatography-quadrupole time-offlight MS to discover the presence of pesticide metabolites in food samples. Journal of Separation Science, 32(13), 2245-2261. Recuperado de https://doi.org/10.1002/jssc. 200900093

Jawaid, S., Talpur, F. N., Nizamani, S. M., Khaskheli, A. A., \& Afridi, H. I. (2016). Multipesticide residue levels in UHT and raw milk samples by GC- $\mu$ ECD after QuEChER extraction method. Environmental Monitoring and Assessment, 188(4), 230. Doi: https:// doi.org/10.1007/s10661-016-5222-6

Lacina, O., Urbanova, J., Poustka, J., \& Hajslova, J. (2010). Identification/quantification of multiple pesticide residues in food plants by ultra-high-performance liquid chromatography-time-of-flight mass spectrometry. Journal of Chromatography A, 1217(5), 648-659. Doi: https://doi.org/10.1016/J.CHROMA. 2009.11.098
Lans-Ceballos, E., Lombana Gomez, M., \& Pinedo-Hernández, J. (2018). Residuos de pesticidas organoclorados en leche pasteurizada distribuida en Montería, Colombia. Revista de Salud Pública, 20(2), 208-214. Doi: https:// doi.org/10.15446/rsap.v20n2.51175

Lee, D.-H., Lee, I.-K., Song, K., Steffes, M., Toscano, W., Baker, B. A., \& Jacobs, D. R. (2006). A strong dose-response relation between serum concentrations of persistent organic pollutants and diabetes: results from the National Health and Examination Survey 1999-2002. Diabetes Care, 29(7), 1638-1644. Doi: https://doi. org/10.2337/dc06-0543

Lorenzatti, E. A., Maitre, M. I., \& Lenardon, A. (2003). Evaluación de la contaminación con plaguicidas en productos lácteos. Revista FAVE - Ciencias Veterinarias, 2(1), 56. Recuperado de http://infolactea.com/wp-content/ uploads/2017/08/1386-3656-1-PB.pdf

Martins, J. G., Amaya Chávez, A., Waliszewski, S. M., Colín Cruz, A., \& García Fabila, M. M. (2013). Extraction and clean-up methods for organochlorine pesticides determination in milk. Chemosphere, 92(3), 233-246. Doi: https://doi.org/10.1016/j.chemosphere. 2013.04.008

Muñoz, C. (2005). Niveles de Residuos de Pesticidas Organoclorados en Leche Pasteurizada UHT procedente de la IX Y X Regiones de Chile (Universidad Austral de Chile). Recuperado de http://cybertesis.uach.cl/tesis/uach/2005/ fam $828 \mathrm{n} / \mathrm{doc} / \mathrm{fam} 828 \mathrm{n} . \mathrm{pdf}$

National Institute of Standards and Technology. (2018). NIST Standard Reference Database 1A v17 | NIST. Recuperado de NIST/ EPA/NIH Mass Spectral Library with Search Program Data Version: NIST v17 Software Version: 2.3 website: https://www.nist.gov/ $\mathrm{srd} /$ nist-standard-reference-database-1a-v17

Pastor Ciscato, C. H., Gebara, A. B., \& de Souza Spinosa, H. (2002). Pesticide residues in cow milk consumed in São Paulo City (Brazil). 
Journal of Environmental Science and Health, Part B, 37(4), 323-330. Doi: https://doi. org/10.1081/PFC-120004473

Polgár, L., García-Reyes, J. F., Fodor, P., Gyepes, A., Dernovics, M., Abrankó, L., ... Molina-Díaz, A. (2012). Retrospective screening of relevant pesticide metabolites in food using liquid chromatography high resolution mass spectrometry and accurate-mass databases of parent molecules and diagnostic fragment ions. Journal of Chromatography A, 1249, 83-91. Doi: https:// doi.org/10.1016/J.CHROMA.2012.05.097

Rahman, M. M., Lee, H. S., Abd El-Aty, A. M., Kabir, M. H., Chung, H. S., Park, J.-H., ... Shim, J.-H. (2018). Determination of endrin and $\delta$-keto endrin in five food products of animal origin using GC- $\mu$ ECD: A modified QuEChERS approach to traditional detection. Food Chemistry, 263, 59-66. Doi: https://doi.org/10.1016/J.FOODCHEM. 2018.04.099

Rawash, E.-S. A., Mohamed, G. G., Souaya, E. R., Khalil, L. H., El-Chaghaby, G. A., El-Gammal, M. H., ... El-Gammal, M. H. (2018). Distribution and Health Hazards of Polycyclic Aromatic Hydrocarbons in Egyptian Milk and Dairy-Based Products. Beverages, 4(3), 63. Doi: https://doi.org/10.3390/beverages4030063

Rodríguez, M., De Pratt, C., Peña, C., \& Beltré, B. (2006). Determinación de pesticidas organoclorados en los vegetales: brócoli, lechuga y apio del Valle de Constanza. (Tesis para la opción del Grado de Licenciatura en Química, Escuela de Química, Facultad de Ciencias, Universidad Autónoma de Santo Domingo, UASD). Recuperado de https://docplayer. es/67500237-Base-de-datos-de-compuestos-organicos-persistentes-cop-s-en-la-republica-dominicana.html

Sbriz, L., Aquino, M. R., Alberto De Rodriguez, N. M., Fowler, S. W., \& Sericano, J. L. (1998). Levels of chlorinated hydrocarbons and trace metals in bivalves and nearshore sediments from the Dominican Republic. Marine Pollution Bulletin, 36(12), 971-979. Doi: https:// doi.org/10.1016/S0025-326X(98)00097-6

Secretaría de Estado de Medio Ambiente y Recursos Naturales (SEMARENA). (2008). Plan nacional de implementación del Convenio de Estocolmo en la República Dominicana $=$ Nacional implementation Plan for the Sockholm Convention in the dominican Republic. Recuperado de Secretaría de Estado de Medio Ambiente y Recursos Naturales (SEMARENA), Programa de las Naciones Unidas para el Desarrollo (PNUD) website: https://opacbiblioteca.intec.edu.do/cgi-bin/ koha/opac-

Secretariat of the Stockholm Convention. (2017). Listing of POPs in the Stockholm Convention. Retrieved May 27, 2019, from All POPs listed in the Stockholm Convention website: http://chm.pops.int/TheConvention/ ThePOPs/AllPOPs/tabid/2509/Default.aspx

Secretariat of the Stockholm Convention. (2019). The POPs. Recuperado de What are POPs? website: http://chm.pops.int/TheConvention/ ThePOPs/tabid/673/Default.aspx

Selvi, C., Paramasivam, M., Rajathi, D. S., \& Chandrasekaran, S. (2012). Multiresidue Analysis of Organochlorine Pesticides in Milk, Egg and Meat by GC-ECD and Confirmation by GC-MS. Bulletin of Environmental Contamination and Toxicology, 89(5), 1051-1056. Doi: https://doi.org/10.1007/s00128-0120789-2detail.pl?biblionumber $=113972 \& s-$ helfbrowse_itemnumber $=90454$

Subsecretaría de Estado de Recursos Naturales de la Secretaría de Estado de Agricultura

(SEA-SURENA). (1999). Planificación y manejo ambiental del litoral de Santo Domingo: plan de saneamiento ambiental para las cuencas media baja Ozama-Isabela-Haina. (P. SEA, Ed.). Recuperado de https://catalogobiblioteca.unapec.edu.do/cgi-bin/koha/opac-detail.pl?biblionumber $=6614$ 
United States Environmental Protection Agency (US EPA), O. (2020). Regulation of Pesticide Residues on Food. Recuperado de https:// www.epa.gov/pesticide-tolerances

Wiley. (2016). Wiley Registry of Mass Spectral Data, 11th Edition | Mass Spectrometry | Analytical Chemistry | Chemistry | Subjects | Wiley. In Mass Spectrometry (11th edition). Recuperado de https://www.wiley.com/en-us/ Wiley+Registry+of+Mass+Spectral+Data\%2C+11th+Edition-p-9781119171010
World Health Organization (WHO) Persistent organic pollutants (POPs). (2014). WHO. Recuperado de https://www.who.int/foodsafety/areas_work/chemical-risks/pops/en/ 